\title{
Wolf-Rüdiger Schenke \\ Das »gefühlte« Misstrauen Zur Verfassungsrechtslage nach der Entscheidung des Bundesverfassungsgerichts vom 25.8.2005 zur Vertrauensfrage nach Art. 68 GG
}

\section{Einfübrung in die Problematik}

Der Pulverdampf hat sich verzogen. Die Auflösung des Bundestags wurde durch das Bundesverfassungsgericht gebilligt. Neuwahlen haben stattgefunden, wenn auch mit einem anderen Ergebnis als von den meisten politischen Akteuren und Beobachtern erwartet. Es ist Zeit, Bilanz zu ziehen. Sicher nicht in dem Sinn, den Streit um die Berechtigung der Auflösung wieder aufleben zu lassen. Die Auflösung des Bundestags ist res judicata und Geschichte: Roma locuta, causa finita. Ohnehin verbietet sich eine argumentative Wiederaufnahme des Verfahrens durch den Autor, der als Prozessvertreter eines der Antragsteller an dem verfassungsgerichtlichen Verfahren unmittelbar beteiligt war und sich als solcher nur zu leicht dem Verdacht aussetzen würde, ein schlechter Verlierer zu sein. Eine Wiederholung der Argumente, die in der Antragsschrift und in der mündlichen Verhandlung zur Begründung der Verfassungswidrigkeit der Auflösungsverfügung angeführt wurden, erübrigt sich ohnehin, weil sie in dem Minderheitsvotum des Bundesverfassungsrichters Jentsch ${ }^{1}$ ihren Niederschlag gefunden haben. Sie sind insoweit auch für die Öffentlichkeit »aktenkundig«. So kann es nur noch darum gehen, in die Zukunft zu schauen und die rechtlichen und politischen Folgen dieser Entscheidung aufzuzeigen. Sie weisen in der Tat weit über den aktuellen Fall hinaus und entfalten insoweit unübersehbare Signalwirkungen für die künftige Handhabung des Art. 68 GG. Diese Folgen müssen den Staatsrechtslehrer von Berufs wegen interessieren. Das gilt speziell dann, wenn er die hier maßgebliche Vorschrift in einem Grundgesetzkommentar zu erläutern hat ${ }^{2}$ und sich schon aus diesem Grund einer rechtlichen Stellungnahme nicht zu entziehen vermag. Deshalb habe ich mich nach anfänglichem Zögern auf die Bitte der Schriftleitung dieser Zeitschrift hin entschieden, zur Verfassungsrechtslage nach der Entscheidung des BVerfG vom 25.08.2005 Stellung zu nehmen. Dies geschieht im Bewusstsein, dass eine solche Folgenerörterung nicht möglich ist, ohne auf jene tragenden Gründe der bundesverfassungsgerichtlichen Entscheidung einzugehen, denen losgelöst von dem konkret entschiedenen Einzelfall weitreichen-

1 BVerfG, NJW 2005, S. $2676 \mathrm{ff}$.

2 S. Wolf-Rüdiger Schenke, in: Bonner Kommentar zum GG (Zweitbearbeitung), Art. 68, Rdnrn. 1 ff. 
de Bedeutung für die Zukunft zukommt. Ein solches Eingehen erfährt seine Rechtfertigung nicht zuletzt daraus, dass die Entscheidung des BVerfG vom 25.08.2005 durchaus neue, in der Art der Begründung nicht voraussehbare Weichenstellungen vorgenommen hat, zu denen bisher noch nicht Stellung genommen werden konnte.

In der Tat markiert das Urteil des BVerfG vom 25.08.2005 einen tiefen Einschnitt in der Verfassungsentwicklung, die das Grundgesetz seit 1949 genommen hat. Dieser Einschnitt ist weit tiefer und von grundsätzlicherer Bedeutung als so manche der zahlreichen Verfassungsänderungen, die das GG seit seinem Inkrafttreten erfahren hat. Der Verfassungswandel, der sich hier in der Judikatur anbahnt, wird freilich bei oberflächlicher Betrachtung nicht offenbar, weil sich das BVerfG zur Begründung seiner Entscheidung auf sein Urteil von 1983 beruft, in der es über die Verfassungsmäßigkeit der Auflösung des Bundestags aufgrund einer vorangegangenen unechten, d. h. mit dem Ziel einer Bundestagsauflösung gestellten Vertrauensfrage des seinerzeitigen Bundeskanzlers Helmut Kohl zu befinden hatte und diese gleichfalls als verfassungsmäßig befand. Damit wird der Eindruck erweckt, das neue Urteil des BVerfG liege ganz auf der Linie seiner bisherigen Rechtsprechung bzw. setze diese nur fort und stelle sich allenfalls als deren Konkretisierung dar. Tatsächlich verändert die neue Judikatur jedoch das Verfassungsgefüge des GG in schwerwiegender Weise, indem sie die verfassungsgerichtliche Kontrolle der Auflösungsentscheidung des Bundespräsidenten weiter zurücknimmt und damit die Normativität der Verfassung schwächt. Eng hiermit zusammenhängend bietet sie eine bisher nicht bestehende Handhabe, das bewusst repräsentativ-demokratische System des Grundgesetzes mit in dieser Weise bisher fremden plebiszitär-demokratischen Elementen politisch anzureichern und damit zu schwächen, neue Akzente bei der Bestimmung des grundgesetzlichen Föderalismus zu setzen und vor allem die Machtbalance zwischen Bundeskanzler und Bundestag erheblich zu verändern.

\section{Materiellrechtliche Grenzen einer unechten Vertrauensfrage}

Schon vor der Entscheidung des BVerfG vom 25.08.2005 bestand weitgehende Einigkeit, dass eine Vertrauensfrage nach Art. 68 GG, die das erklärte Ziel verfolgt, den Bundestag zur Verneinung des Vertrauens zu veranlassen, um auf diese Weise seine Auflösung zu ermöglichen (sogenannte unechte Vertrauensfrage), nur unter sehr eingeschränkten Voraussetzungen verfassungsrechtlich zulässig ist. Der Bundeskanzler sollte »dieses Verfahren nur dann anstrengen dürfen, wenn es für ihn politisch nicht mehr gewährleistet ist, mit den im Bundestag bestehenden Kräfteverhältnissen weiterzuregieren $\aleph^{3}$, d. h. wenn er die für die Verfolgung und Durchsetzung seiner Politik erforderliche Mehrheit im Bundestag verloren hat. Als Begründung für diese Einschränkung des Art. 68 GG wurden in der Rechtsprechung des BVerfG, aber auch in der Verfassungsrechtslehre eine Reihe systematischer und teleologischer Gesichtspunkte aufgeführt ${ }^{4}$. Die wichtigsten waren: Ohne eine ent-

3 BVerfGE 62, S. 1 (2, Leitsatz 6).

ZfP 53. Jg. $1 / 2006$ 
sprechende Einschränkung würde die Entscheidung des Grundgesetzes für eine grundsätzlich vierjährige Amtszeit in Frage gestellt (Art. 38 Abs. 1 S. 2 GG in Verbindung mit Art. 39 Abs. 1 S. 1 GG), das Bekenntnis des Grundgesetzes für ein bewusst repräsentativ-demokratisches System würde durch Anreicherung mit plebiszitär-demokratischen Elementen ausgehöhlt, die Chancengleichheit der politischen Parteien würde durch die Chance zur Bestimmung des Wahltermins beeinträchtigt und die konstitutionelle Stellung des Bundespräsidenten verändert, der nunmehr nicht nur in engbegrenzten Ausnahmefällen zum politischen Akteur würde. Schliesslich liefe eine Auflösung des Bundestags faktisch auf ein dem Grundgesetz fremdes Auflösungsrecht des Bundeskanzlers hinaus und schwächte das parlamentarische Regierungssystem, wie es in Art. 67 GG seinen Ausdruck gefunden hat.

\section{Der Beurteilungsspielraum des Bundeskanzlers bei der Stellung einer unechten Vertrauensfrage}

Das eigentliche Problem, das sich hierbei stellt, bestand und besteht freilich in praxi darin, die Fälle, in denen ein Einsatz des verfassungsrechtlichen Instrumentariums des Art. 68 GG zulässig ist, von jenen abzugrenzen, bei denen die Stellung der Vertrauensfrage und die sich hieran anschliessende Prozedur verfassungswidrig sind, weil der Kanzler nach wie vor für seine Politik eine ausreichende parlamentarische Unterstützung besitzt. Der gelegentlich unternommene Versuch, diese Problematik durch die Bejahung der generellen Verfassungswidrigkeit einer unechten Vertrauensfrage zu entschärfen, vermochte nicht zu überzeugen. Dem Kanzler, der offensichtlich das Vertrauen des Parlaments verloren hat, muss die Möglichkeit eingeräumt werden, eine unechte Vertrauensfrage zu stellen. Wenn für ihn keine Chance besteht, eine Mehrheit zu erlangen, kann ihm nicht zugemutet werden, den mit ei-

4 Siehe dazu ausführlich Schenke, in: Bonner Kommentar zum Grundgesetz (Zweitbearbeitung), Art. 68, Rdnrn. 62 ff.; ders., Die verfassungswidrige Bundestagsauflösung, NJW 1982, S. 2521 ff.; Volker Epping, in: Hermann v. Mangoldt / Friedrich Klein / Christian Starck, Grundgesetz, Bd. II, 5. Aufl. 2005, Rdnrn. 12 ff.; Georg Hermes, in: Horst Dreier, Grundgesetz, Bd. II, 1998, Art. 68, Rdnrn. 10 ff.; Ute Mager, in: Ingo v. Münch / Philip Kunig, Grundgesetz Bd. II, 5. Aufl. 2001, Art. 68, Rdnrn. 9 ff.; Martin Oldiges, in: Michael Sachs, Grundgesetz, 3. Aufl. 2003, Art. 68, Rdnrn. 14 f.; Klaus Stern, Das Staatsrecht der Bundesrepublik Deutschland, Bd. I, 2. Aufl. 1984, $\mathbb{S} 22$ III, 3b. Speziell zur Frage der Verfassungsmäßigkeit der Bundestagsauflösung 2005 Jörn Ipsen, Zur Regierung verurteilt? - Verfassungsrechtliche Probleme der Vertrauensfrage nach Art. 68 GG, NJW 2005, S. 2201 ff.; ders., Die Auflösung des 15. Deutschen Bundestages - eine Nachlese, NVwZ 2005, S. 1147 ff.; Wolfgang Löwer, Inszeniertes Misstrauen, DVBl. 2005, S. 1102 ff.; Christian Pestalozza, Art. 68 GG light oder Die Wildhüter der Verfassung, NJW 2005, S. 2817 ff.; Bernd Pfeifer, Bundestagsauflösung trotz Kanzlermehrheit?, VR 2005, S. 253 ff.; Franz Reimer, Vertrauensfrage und Bundestagsauflösung bei parlamentarischer Anscheinsgefahr, JuS 2005, S. 680 ff.; WolfRüdiger Schenke / Peter Baumeister, Vorgezogene Bundestagswahlen: Überraschungscoup ohne Verfassungsbruch?, NJW 2005, S. 1844 ff.; Christian Starck, Anmerkung zu BVerfG, Urteil vom 25.08.2005, JZ 2005, S. 1053 ff.; Jörg Philipp Terhechte, Die vorzeitige Bundestagsauflösung als verfassungsrechtliches Problem, Jura 2005, 512 ff. 
ner Selbstbeschädigung verbundenen, von vorneherein untauglichen und überdies unehrlichen Versuch zu unternehmen, das Parlament zu einer Bekundung des Vertrauens aufzufordern.

Die zentrale Aufgabe, vor die sich das BVerfG bei seiner Entscheidung vom 25.08.2005 ähnlich wie bei seiner Entscheidung vom 16.02.1983 gestellt sah, bestand damit darin, Kriterien zu entwickeln, anhand derer festgestellt werden kann, ob der Bundeskanzler für seine Politik im Parlament über eine ausreichende Mehrheit verfügt, und anhand dieser den konkreten Einzelfall zu beurteilen. Im Hinblick auf die Schwierigkeit, die mit einer solchen Einschätzung verbunden ist, hatte das BVerfG in seiner Entscheidung aus dem Jahre 1983 dem Bundeskanzler einen durch die anderen Verfassungsorgane, insbesondere auch durch den Bundespräsidenten und das BVerfG, zu respektierenden Beurteilungsspielraum eingeräumt. Danach war die Einschätzungs- und Beurteilungskompetenz des Kanzlers dann zu respektieren, wenn eine andere Einschätzung der politischen Lage der Einschätzung des Bundeskanzlers nicht eindeutig vorzuziehen war. Gerechtfertigt wurde diese Einräumung eines auch durch das BVerfG zu respektierenden Beurteilungsspielraums in den Entscheidungen sowohl von 1983 wie auch von 2005 damit, dass drei Verfassungsorgane - der Bundeskanzler, der Deutsche Bundestag und der Bundespräsident - es jeweils in der Hand hätten, die Auflösung nach ihrer freien politischen Einschätzung zu verhindern, und dass dies dazu beitrage, die Verlässlichkeit der Annahme zu sichern, die Bundesregierung habe ihre parlamentarische Handlungsfähigkeit verloren ${ }^{5}$.

\section{Neue Weichenstellungen des Bundesverfassungsgerichts in Verbindung mit der Überprüfung einer unechten Vertrauensfrage}

Mit neuen Fragen sah sich das BVerfG bei der Beurteilung der unechten Vertrauensfrage Schröders insoweit konfrontiert, als die Vertrauensfrage dadurch begründet wurde, dass der Kanzler für seine Politik eine neue Legitimation durch den demokratischen Souverän benötige (dazu 1.), die Mehrheitsverhältnisse im Bundesrat ihn zur Stellung der unechten Vertrauensfrage zwängen (dazu 2.) und die Vertrauensfrage trotz eines nicht offen zutage getretenen, durch den Bundeskanzler aber behaupteten Verlustes seiner politischen Handlungsfähigkeit erfolgte (dazu 3.). Dabei schien das BVerfG bei seiner Stellungnahme zu diesen Fragen davon ausgegangen zu sein, dass, falls einer der vom Kanzler angegebenen tragenden Gründe einer verfassungsrechtlichen Prüfung nicht standhielte, allein dies schon die Verfassungswidrigkeit der unechten Vertrauensfrage nach sich ziehen müsste. Anders lässt sich jedenfalls der erhebliche argumentative Aufwand, den das Gericht in bezug auf die Begründung der Vertrauensfrage durch eine vom Kanzler gewollte Legitimation seiner Reformpolitik sowie die bestehende »obstruierende « Bundesratsmehrheit entfaltete, schwerlich erklären. Damit knüpfte das BVerfG - trotz sich hier stellen-

5 So Leitsatz 4 c der Entscheidung vom 25.08.2005 und ähnlich Leitsatz 9 der Entscheidung vom 16.02.1983. 
der prinzipieller Bedenken m. E. im Ergebnis wohl zu Recht - an allgemeine Grundsätze der Ermessenslehre an. Danach ist dann, wenn sich einer der tragenden Gründe einer kumulativ begründeten Ermessensentscheidung als rechtswidrig erweist, die Entscheidung insgesamt rechtswidrig.

\section{Die Begründung der Vertranensfrage unter Hinweis auf die Notwendigkeit einer Legitimation der Regierungspolitik durch den demokratischen Souverän}

Ausgangspunkt für die verfassungsrechtliche Bewertung des Verhaltens des Bundeskanzlers mussten dessen Begründungen für die Stellung der Vertrauensfrage sein. Das war schon deshalb unumgänglich, weil nicht nach außen getretene innere Motivationen sich naturgemäß einer rechtlichen Würdigung entziehen. Der Bundeskanzler musste sich damit beim Wort nehmen lassen und seine Worte wohl wägen, wenn überhaupt eine rechtliche Überprüfung der von ihm gestellten Vertrauensfrage im Hinblick darauf möglich sein sollte, ob die durch das BVerfG schon in seiner Entscheidung von 1983 aufgestellten Voraussetzungen für die Stellung einer unechten Vertrauensfrage gegeben waren. Er durfte deshalb die Begründung der Vertrauensfrage nicht auf Argumente stützen, die im diametralen Gegensatz zur Rechtsprechung des BVerfG stehen. Zur Begründung einer solchen Verpflichtung bedurfte es weder eines Rückgriffs auf allgemeine Ermessensgrundsätze noch der Bemühung des allgemeinen ungeschriebenen Grundsatzes der Verfassungsorgantreue $^{6}$, der die Verfassungsorgane zu gegenseitigem Respekt verpflichtet. Eine entsprechende Verpflichtung ergab sich vielmehr bereits aus $₫ 31$ BVerfGG, der nach der ständigen Rechtsprechung des BVerfG alle staatlichen Organe dazu verpflichtet, sich nicht in Widerspruch zu tragenden Gründen der verfassungsgerichtlichen Rechtsprechung zu setzen ${ }^{7}$. Von daher gesehen erweckte es verfassungsrechtliche Bedenken, wenn der Bundeskanzler schon in seiner Ankündigung der Vertrauensfrage, bei der ihm nach der Rechtsprechung des BVerfG bereits eine Überprüfung der rechtlichen Voraussetzungen für die Antragstellung oblag8, ausführte, »für die aus meiner Sicht notwendige Fortführung der Reformen halte ich eine klare Unter-

6 Dazu eingehend Wolf-Rüdiger Schenke, Die Verfassungsorgantreue, 1977 sowie Ralph Lorz, Interorganrespekt und Verfassungsrecht, 2001; auch das BVerfG geht in ständiger Rechtsprechung von der Existenz eines solchen dem Prinzip der Bundestreue vom verfassungstheoretischen Ansatzpunkt eng verwandten Rechtsprinzips aus, vgl. z. B. BVerfGE 94, S. 166 (234).

7 Dazu z. B. BVerfGE 1, S. 14 (36 f.); 20, S. 56 (87); 40, S. 88 (93); dazu Wolfgang Hoffmann-Riem, Beharrung oder Innovation - zur Bindungswirkung verfassungsgerichtlicher Entscheidungen, Der Staat Bd. 13 (1974), S. 335 ff.; Michael Sachs, Die Bindungen des BVerfG an seine Entscheidungen, 1977, S. 66 ff.; Schenke, Verfassungsgerichtsbarkeit und Fachgerichtsbarkeit, 1987, S. $60 \mathrm{ff}$.

8 S. dazu BVerfGE 62, S. 1 (49 f.): »Ob die Kräfteverhältnisse im Bundestag eine Lage aufweisen, die eine vom stetigen Vertrauen der Mehrheit getragene und unterstützte Politik des Kanzlers nicht mehr sinnvoll ermöglicht, ... hat der Bundespräsident bereits zu prüfen, wenn er beabsichtigt, einen Antrag mit dem Ziel zu stellen, darüber die Auflösung des Bundestages anzustreben«. 
stützung durch eine Mehrheit der Deutschen gerade jetzt für erforderlich «" Auch im folgenden spielte die Notwendigkeit, das Volk über die Grundrichtung der Politik, insbesondere die Hartz-IV-Gesetze, entscheiden zu lassen, eine entscheidende Rolle bei der Begründung. So rechtfertigte der Bundeskanzler die Stellung der Vertrauensfrage vor dem Bundestag mit dem Hinweis, »wenn diese Agenda fortgesetzt werden und weitergeführt werden soll - und das muss sie - , ist eine Legitimation durch Wahlen unverzichtbar ${ }^{10}$, und legte im folgenden dar: »Vordergründig betrachtet handelt es sich um einen Vorgang, in dem der Bundeskanzler sein eigenes Schicksal der Entscheidung des Volkes anvertraut. Die wahre Dimension unserer heutigen Entscheidung weist aber weit darüber hinaus. Tatsächlich geht es um die Möglichkeit des demokratischen Souveräns, die Grundrichtung der Politik selbst zu bestimmen ${ }^{11}$. Hier wie auch an weiteren Äußerungen des Bundeskanzlers, die er im Rahmen seiner Begründung der Vertrauensfrage vor dem Bundestag wie auch an anderer Stelle abgab, tritt mit aller Deutlichkeit hervor, dass der Zweck der Vertrauensfrage primär, jedenfalls aber zu einem wesentlichen Teil in einer plebiszitären Bestätigung der mit dem Begriff der Agenda 2010 gekennzeichneten Reformpolitik und damit in der Verfolgung einer Zielsetzung lag, die nach der Entscheidung des BVerfG aus dem Jahre 1983 und der herrschenden Staatsrechtslehre gerade nicht mehr durch Art. 68 GG gedeckt ist.

Unter diesen Umständen ist es unverständlich, wenn das BVerfG ein wichtiges, wenn nicht sogar das zentrale Begründungselement bei der Stellung der Vertrauensfrage herunterspielte, indem es behauptete, »eine solche Formulierung kann als rhetorische Floskel gemeint sein, die eine Referenz an das Demokratieprinzip zum Ausdruck bringt «, und im selben Atemzug behauptete, »es lässt sich auch nicht feststellen, dass der Kanzler ein dem Zweck des Art. 68 GG widersprechendes Plebiszit anstrebt ${ }^{12}$. Rhetorische Gründe legitimieren nicht zur verbalen und inhaltlichen Missachtung verfassungsrechtlicher und verfassungsgerichtlicher Vorgaben, und deutlicher, als durch den Kanzler geschehen, konnte auch nicht klargestellt werden, dass es um ein Votum des Volkes über das mit dem Stichwort Agenda 2010 umschriebene Reformpaket ging.

Dass darüber hinaus die Autorität des BVerfG Schaden zu nehmen droht, wenn es einen solchen Affront eines anderen Verfassungsorgans duldet, lässt sich nicht von der Hand weisen. Vor allem wird durch die Zulassung solcher »rhetorischer Floskeln « die Hemmschwelle für den Einsatz der Vertrauensfrage erheblich gesenkt, indem der Kanzler das Eingeständnis des Scheiterns seiner Politik im Bundestag und des Verlusts der politischen Mehrheit durch Hinweis auf sein Bestreben, den Souverän über seine Politik entscheiden zu lassen, »demokratisch « zu bemänteln vermag. Zudem fördert eine gerichtlich nicht gerügte Zulassung einer solchen Begründung bei der Bevölkerung den Eindruck, hier läge eine verfassungsrechtlich

9 S. http://www.n24.de/index.php?n2000505222352117000002.

10 Stenografischer Bericht über die 185. Sitzung der 15. Wahlperiode, S. 17465 (B).

11 Stenografischer Bericht, a.a.O., S. 17468 (B).

12 Urteil des BVerfG vom 25.08.2005, http://www.bverfg.de/.entscheidungen/es20050825 _2bve000405.html, Rdnrn. 177 f. = NJW 2005, S. 2669 (2675). 
zulässige Begründung einer Vertrauensfrage vor. Die Problematik einer solchen Herabstufung der Begründung der Vertrauensfrage, wie sie in ihrer »Nichternstnahme « liegt, wird zusätzlich daran deutlich, dass gerade dann, wenn einem Verfassungsorgan bei der Bewertung eines politischen Sachverhalts ein Beurteilungsspielraum eingeräumt wird, der Begründung seiner Entscheidungen im Einklang mit sonst allgemein anerkannten Grundsätzen bei deren gerichtlicher Überprüfung eine besondere Bedeutung eingeräumt werden muss.

\section{Die Berufung auf die Mehrbeitsverbältnisse im Bundesrat}

Deutlich wurde die Unvereinbarkeit der durch den Bundeskanzler gestellten Vertrauensfrage mit dem Urteil des BVerfG aus dem Jahre 1983 auch an einem anderen tragenden, durch den Bundeskanzler zur Legitimation seiner Vertrauensfrage angeführten Argument. Diese rechtfertigte er auch damit, dass »nur eine durch die Wählerinnen und die Wähler klar und neuerlich legitimierte Regierungspolitik ... bei der Mehrheit des Bundesrats zu einem Überdenken der Haltung und - wenn auch nicht kurzfristig - zu einer Änderung der Mehrheit führen « werde ${ }^{13}$. Das ließ sich offensichtlich nicht damit in Einklang bringen, dass es nach der früheren Judikatur des BVerfG nur darauf ankommen sollte, dass der Bundeskanzler das Vertrauen der Mehrheit der Mitglieder des Bundestags verloren hat. Nur wenn ein solcher Vertrauensverlust vorliege, sei eine (unechte) Vertrauensfrage mit dem Ziel der Herbeiführung von Neuwahlen zulässigi ${ }^{14}$. Das BVerfG ${ }^{15}$ führte in diesem Zusammenhang aus: »Der Bundeskanzler, der die Auflösung des Bundestags auf dem Weg des Art. 68 GG anstrebt, soll dieses Verfahren nur (Kursivdruck im Original) anstrengen dürfen, wenn es politisch nicht mehr gewährleistet ist, mit den im Bundestag bestebenden Kräfteverbältnissen (Kursivdruck durch Verfasser) weiter zu regieren«.

War damit die auf die Mehrheitsverhältnisse im Bundesrat abstellende Argumentation des Bundeskanzlers schon aus diesem Grund verfassungsrechtlich nicht haltbar und ungeeignet, eine unechte Vertrauensfrage zu rechtfertigen, so kam noch ein anderer Gesichtspunkt hinzu: Auch wenn die Wähler die Politik der Regierung bestätigt und deren parlamentarische Basis vergrößert hätten (was ja bekanntlich nicht geschah), hätte sich an den Mehrheitsverhältnissen im Bundesrat und den hieraus resultierenden Schwierigkeiten bei der Durchsetzung der Regierungspolitik sowie der Notwendigkeit, politische Kompromisse einzugehen, zunächst gar nichts geändert. Das wurde bezeichnenderweise auch vom Bundeskanzler konzediert, indem er darauf hinwies, dass das Wählervotum jedenfalls kurzfristig nicht zu einer Änderung der Mehrheit im Bundesrat führen werde. Auch der Umstand, dass eine neue

13 Stenografischer Bericht, a.a.O., S. 17467 (D).

14 Dazu, dass die unechte Vertrauensfrage kein Instrument darstellt, das zur Behebung eines politischen Konflikts zwischen Bundestags- und Bundesratsmehrheit geeignet ist, s. auch Wolf-Rüdiger Schenke, Gesetzgebung zwischen Parlamentarismus und Föderalismus, in: Hans-Peter Schneider / Wolfgang Zeh, Parlamentsrecht und Parlamentspraxis in der Bundesrepublik Deutschland, 1989, S. 1485 (1505, Rdnr. 42).

15 BVerfGE 62, S. 1 (2, Leitsatz 6). 
Legitimation der Regierungspolitik durch den Wähler es der Bundesratsmehrheit politisch erschweren würde, eine vom Kanzler behauptete Obstruktionspolitik zu betreiben, rechtfertigte es nicht, über eine unechte Vertrauensfrage auf die Auflösung des Bundestags hinzuwirken. Würde dies für die Stellung einer unechten Vertrauensfrage bereits ausreichen, so ergäbe sich immer dann, wenn die Bundestagsopposition im Bundesrat die Mehrheit stellt - und dies trifft, einmal abgesehen von kurzen Unterbrechungen, seit Jahrzehnten zu - die Möglichkeit, eine unechte Vertrauensfrage mit dem Ziel zu stellen, auf diese Weise eine Auflösung des Bundestags und Neuwahlen zu initiieren. Die Gefährdung der politischen Stabilität wäre bei einer in so weitem Umfang eröffneten Möglichkeit zur Stellung einer unechten Vertrauensfrage und einer hierdurch eröffneten Flucht des Kanzlers und der ihn unterstützenden Bundestagsabgeordneten aus der politischen Verantwortung evident.

Der vom Bundeskanzler in einem Schreiben an den Bundespräsidenten vom 12.07.2005 unternommene und durch das BVerfG akzeptierte Versuch, eine Beziehung zwischen den Mehrheitsverhältnissen im Bundesrat und der politischen Handlungsfähigkeit des Bundeskanzlers unter Hinweis darauf zu begründen, dass es angesichts der Mehrheitsverhältnisse im Bundesrat erschwert werde, die Geschlossenheit der Koalitionsmehrheit im Bundestag sicherzustellen und damit politische Vorgaben in einer ohnehin knappen Parlamentsmehrheit durchzusetzen ${ }^{16}$, vermag demgegenüber nicht zu überzeugen. Solche Schwierigkeiten sind in der grundgesetzlich vorgegebenen Stellung des Bundesrats und seinem damit begründeten politischen Mitspracherecht angelegt, können damit aber als solche noch nichts zur Legitimation einer unechten Vertrauensfrage beitragen. Zwar ist es bei knappen Mehrheitsverhältnissen zugegebenermaßen schwieriger, einen mit der Mehrheit der Stimmen des Bundesrats beschlossenen Einspruch zurückzuweisen, da dies nach Art. 77 Abs. 4 S. 1 GG nur mit den Stimmen der Mehrheit der Mitglieder des Bundesrats möglich ist. Dieser allgemeine Gesichtspunkt vermag aber allein gesehen die Stellung einer Vertrauensfrage noch nicht zu rechtfertigen. Wenn man dies bereits für ihre Legitimation ausreichen ließe, bedeutete dies nichts anderes, als dass bei der Beurteilung der Kräfteverhältnisse entgegen den verfassungsgerichtlichen Vorgaben aus dem Jahr 1983 eben doch auf die Mehrheitsverhältnisse nicht nur im Bundestag, sondern auch im Bundesrat abgestellt würde.

Deshalb wäre es unumgänglich gewesen, eine Einschränkung der politischen Handlungsfähigkeit des Bundeskanzlers durch eine »Obstruktionspolitik « des Bundesrats jedenfalls zumindest dadurch zu belegen, dass die Verabschiedung eines für die Regierungspolitik wichtigen Gesetzes daran scheiterte, dass die zur Zurückweisung eines Einspruchs erforderliche Kanzlermehrheit nicht erreicht wurde. Einen solchen Nachweis konnte aber Bundeskanzler Schröder nicht erbringen, da er immer dann, wenn eine Kanzlermehrheit zur Zurückweisung eines Einspruchs erforderlich war, die hierfür erforderliche Mehrheit im Bundestag zu erlangen ver-

16 S. dazu BVerfG, a.a.O. (Fn. 12), Rdnr. 179 = NJW 2005, S. 2669 (2675) und das dort zitierte Schreiben des Bundeskanzleramts an das Bundespräsidialamt vom 12.07.2005, S. $12 \mathrm{f}$. 
mochte. In den 29 Fällen, in welchen der Bundesrat einen Einspruch einlegte und auf die sich der Kanzler berief ${ }^{17}$, wurde immer der Einspruch im Bundestag mit der erforderlichen Mehrheit der Mitglieder des Bundestags (Art. 121 GG), der sogenannten Kanzlermehrheit, zurückgewiesen. Es lagen auch keine konkreten Anhaltspunkte dafür vor, dass dies bei einem zur Verfolgung der Regierungspolitik erforderlichen zukünftigen und durch den Kanzler zu benennenden Gesetz zukünftig nicht gelingen sollte, zumal die politisch umstrittenen Gesetze, derer es zur Durchführung der Agenda 2010 bedurfte, bereits weitgehend verabschiedet worden waren.

Eine auf die Veränderung der Mehrheitsverhältnisse im Bundesrat abzielende Rechtfertigung der unechten Vertrauensfrage und die Tolerierung dieser Begründung durch das BVerfG erweckte aber auch im Hinblick auf das verfassungsrechtlich garantierte Bundesstaatsprinzip zusätzliche Bedenken. Eine derartige Begründung der unechten Vertrauensfrage förderte nämlich die ohnehin schon bestehende Tendenz, Landtagswahlen in erster Linie unter bundespolitischen Aspekten zu führen, noch weiter. Die Landtagswahlen würden damit in zunehmendem Maße zu Bundes(rats)wahlen, so dass das politische Geschehen in den Ländern in den Hintergrund gedrängt und die Landtagswahlen und Landtage bundespolitisch instrumentalisiert würden. Die hieraus erwachsenden Gefahren für die Eigenstaatlichkeit der Länder sind unübersehbar. Damit wird eine Politikverflechtung forciert, in deren Konsequenz sich eine Schwächung des grundgesetzlichen föderalen Systems ergibt. Zudem lief die Argumentation des Bundeskanzlers faktisch-politisch auf ein Wählervotum des Bundesvolks gegen den durch die Landesvölker personell-demokratisch legitimierten Bundesrat hinaus. Eine derartige Delegitimation der Landessouveräne durch den Bundessouverän lässt sich mit der Idee des Bundesstaats nicht in Einklang bringen. Bezeichnenderweise führte denn auch das BVerfG ${ }^{18}$ aus, dass eine Vertrauensfrage, die allein mit dem Ziel geführt würde, den Bundesrat mit einer Bestätigung der Bundesregierung durch Wahlakklamation zu delegitimieren, verfassungsrechtlich unzulässig ist.

\section{Der Verlust der politischen Handlungsfähigkeit im Bundestag im Gefolge einer »verdeckten Minderheitssituation"}

Der einzige Gesichtspunkt, der eine unechte Vertrauensfrage rechtfertigen könnte, wäre unter Zugrundelegung der Entscheidung aus dem Jahre 1983 dann gegeben gewesen, wenn der Bundeskanzler von einem Verlust der politischen Handlungsfähigkeit im Hinblick auf den Verlust der politischen Mehrheit im Bundestag ausgegangen und eine entsprechende Einschätzung nicht eindeutig fehlerhaft gewesen wäre. Nachdem der Bundeskanzler in seiner Ankündigung der Vertrauensfrage noch mit keinem Wort auf diesen für die verfassungsrechtliche Beurteilung einer unechten Vertrauensfrage entscheidenden Gesichtspunkt eingegangen war, stellte er dann in

17 Stenografischer Bericht, a.a.O., S. 17467 (C und D).

I 18 BVerfG, a.a.O. (Fn. 12), Rdnr. 151 = NJW 2005, S. 2669 (2672). 
der Begründung seiner Vertrauensfrage mit darauf ab, dass Vertrauen nicht mehr vorhanden sei. Wörtlich führte er hierzu aus: »Eine Bewertung der politischen Kräfteverhältnisse vor und nach der Entscheidung, Neuwahlen anzustreben, muss dazu führen - dessen bin ich mir ganz sicher - , dass ich unter den aktuellen Bedingungen nicht auf das notwendige, auf stetiges Vertrauen im Sinn des Art. 68 Grundgesetz rechnen kann «19. Freilich blieben seine Begründungen für diese Einschätzung wenig präzise, indem er sich darauf berief, dass »vermehrt abweichende, jedenfalls die Mehrheit gefährdende Stimmen laut geworden ${ }^{20}$ seien und dass auch dort, wo Vertrauen nicht mehr vorhanden gewesen sei, öffentlich so getan worden sei, als gäbe es dieses Vertrauen; dies müsse Gegenstand seiner politischen Bewertung $\operatorname{sein}^{21}$. Damit fehlte es an nachprüfbaren Fakten, die seine Einschätzung belegen konnten. Die Berufung darauf, dass in der laufenden Wahlperiode der Bundesrat in 29 Fällen nach abgeschlossenen Vermittlungsverfahren Einspruch gegen ein entsprechendes Gesetz eingelegt habe und dass es weder der Regierung noch den Regierungsfraktionen zumutbar sei, »immer wieder Konzessionen zu machen und doch $\mathrm{zu}$ wissen, dass die Bundesratsmehrheit ihre destruktive Blockadehaltung nicht aufgeben wird «, hat jedenfalls keinen unmittelbaren Bezug zu den politischen Mehrheitsverhältnissen im Bundestag. Wohl aus dem Gefühl heraus, dass es an ausreichenden Belegen für den Verlust der politischen Handlungsfähigkeit fehle, stellte das Bundeskanzleramt nach der Stellung der Vertrauensfrage dem Bundespräsidenten ein Dossier zur Verfügung, in dem sich kritische Äußerungen von Mitgliedern der Regierungsfraktionen fanden.

\section{a) Die „verdeckte Minderheitssituation des Bundeskanzlers“}

Wie der Bundeskanzler in seiner Begründung der Vertrauensfrage zum Ausdruck gebracht hatte, war ein Bestandteil seiner politischen Bewertung, dass trotz entgegengesetzter öffentlicher Vertrauensbekundungen ein Teil der Abgeordneten der Regierungsfraktionen tatsächlich kein Vertrauen zu ihm habe. Das BVerfG verwandte zur Umschreibung dieses Sachverhalts die Bezeichnung einer »verdeckten Minderheitssituation des Bundeskanzlers $\aleph^{22}$. Sie soll dann gegeben sein, »wenn eine organisierte parlamentarische Mehrheit - die nominelle Kanzlermehrheit - sich zwar zu dem von ihr gewählten Kanzler erklärt und ihm äußerlich politische Unterstützung gewährt, diese Unterstützung seines politischen Kurses aber in Wirklichkeit nicht so wirksam ist, dass der Bundeskanzler die von ihm konzeptionell vertretene Politik durchzusetzen vermag ${ }^{23}$. $\mathrm{Zu}$ dieser verdeckten Minderheitssituation führt das BVerfG, und hierin liegt in der Tat eine wesentliche Neuerung gegenüber der Entscheidung aus dem Jahr 1983, aus: »Eine Erosion und der nicht offen gezeig-

19 Stenografischer Bericht, a.a.O., S. 17467 (C).

20 Stenografischer Bericht, a.a.O., S. 17467 (B).

21 Stenografischer Bericht, a.a.O., S. 17467 (C).

22 BVerfG, a.a.O. (Fn. 12), Rdnr. 154 = NJW 2005, S. 2669 (2672 f.).

23 BVerfG, a.a.O. (Fn. 12), Rdnr. 154 = NJW 2005, S. 2669 (2672 f.). 
te Entzug des Vertrauens lassen sich ihrer Natur nach nicht ohne weiteres in einem gerichtlichen Verfahren darstellen und feststellen. Was im politischen Prozess in legitimer Weise nicht offen ausgetragen wird, muss unter den Bedingungen des politischen Wettbewerbs auch gegenüber anderen Verfassungsorganen nicht vollständig offenbart werden. Die Einschätzung des Bundeskanzlers, er sei für seine künftige Politik nicht mehr ausreichend handlungsfähig, ist eine Wertung, die durch das BVerfG schon praktisch nicht eindeutig und vollständig überprüft werden kann und ohne Beschädigung des politischen Handlungssystems auch nicht den üblichen prozessualen Erkenntnismitteln zugänglich ist. Selbst wenn man eine Beweisaufnahme für möglich und geboten hielte, bliebe dem Kanzler ein Einschätzungsspielraum in Bezug auf festgestellte Tatsachen, insbesondere in Bezug auf deren Bedeutung für die künftige Entwicklung. Dem politischen Willensbildungsprozess mit seinen zulässigen, auch von taktischen und strategischen Motiven geprägten Verhaltensweisen und Rücksichtnahmen darf in Fragen der politischen Einschätzung nicht mit einer nach vollem Beweis strebenden gerichtlichen Sachverhaltsaufklärung Schaden zugefügt werden. Andernfalls wäre die vom Grundgesetz gewollte Balance zwischen effektiver rechtlicher Bindung der öffentlichen Gewalt und der Ermöglichung wirksamer politischer Handlungsfreiheit verletzt $\ll^{24}$.

Bemerkenswert ist an diesen Ausführungen bereits die Verwendung des Begriffs der »verdeckten Minderheitssituation des Bundeskanzlers«. Dieser Terminus ist nicht unproblematisch. Artikuliert sich nämlich das Vertrauen des Bundestags zum Bundeskanzler in Abstimmungsmehrheiten im Bundestag, welche die Zustimmung $\mathrm{zu}$ dessen Person und Politik signalisieren, so erscheint es zweifelhaft, ob es überhaupt so etwas wie eine »verdeckte Minderheitssituation« geben kann. Jedenfalls ist die Benutzung dieser Begrifflichkeit durch das BVerfG zumindest deshalb fragwürdig, weil das Gericht dabei implizit davon ausgeht, es könne an einem Vertrauen i. S. des Art. 68 GG fehlen, obwohl sich der Vertrauensverlust nach außen hin gar nicht offenbart. Damit wird aber etwas unterstellt, was es noch zu beweisen gilt, so dass der Vorwurf eines Zirkelschlusses nicht fern liegt. Noch problematischer wird es, wenn das Gericht sich bei einer »verdeckten Minderheitssituation des Bundeskanzlers « nicht oder allenfalls sehr begrenzt für befugt erachtet, in eine Prüfung der Umstände einzutreten, aus welchen sich die »verdeckte Minderheitssituation « ergeben soll. Das begründet nicht nur die Gefahr, dass all jene Akte, in denen sich nach außen hin die Zustimmung zu Person und Politik des Bundeskanzlers manifestiert da für die »verdeckte Minderheitssituation « charakteristisch - bei der politischen Bewertung ausgeklammert oder jedenfalls in ihrem Beweiswert heruntergespielt werden ${ }^{25}$. Es impliziert darüber hinaus, dass ausgerechnet in einer solchen »verdeckten Minderheitssituation des Bundeskanzlers«, bei welcher eine verfassungsge-

| 24 BVerfG, a.a.O. (Fn. 12), Rdnr. 154 = NJW 2005, S. 2669 (2672 f.).

25 Bezeichnend ist dafür etwa, wenn das BVerfG dem Umstand keinerlei Beachtung schenkt, dass es bei Gesetzen, die in der Vergangenheit auch innerhalb der Regierungsfraktionen politisch höchst umstritten waren - wie dem sogenannten Hartz-IV-Gesetz - dennoch zu einer fast einstimmigen Verabschiedung - bis auf eine Stimmenthaltung durch die Regierungsfraktionen kam. 
richtliche Überprüfung der vom Kanzler behaupteten politischen Handlungsunfähigkeit in besonderer Weise angezeigt erscheint, die verfassungsgerichtliche Kontrolle zurückgenommen und letztlich aufgegeben wird.

Der Hinweis des BVerfG, auch bei einer »verdeckten Minderheitssituation« müsse die Bewertung des Kanzlers »auf Tatsachen gestützt sein «²6, vermag diesen Einwand nicht zu entkräften, wenn diese »Tatsachen« in der Logik der »verdeckten Minderheitssituation « ausschließlich Vorgänge im außerparlamentarischen Raum (wie den Verlust von Landtagswahlen, kritische Äußerungen von Politikern der Regierungsfraktionen und Spekulationen über die Gründe für den Wechsel im Parteivorsitz) betreffen, sie überdies »kanzlergetönt« interpretiert und auf eine höchst selektive Weise ermittelt werden, d. h. wesentliche Gesichtspunkte ausgeklammert bleiben, die gegen den Verlust der parlamentarischen Handlungsfähigkeit sprechen könnten (dazu auch unten IV. 3.). Man denke in diesem Zusammenhang nur an das im Mehrheitsvotum nicht einmal erwähnte Wahlmanifest der SPD vom 04.07.2005, in dem bekräftigt wurde, dass die »Agenda 2010 « konsequent umgesetzt werden solle ${ }^{27}$, oder an die öffentlichen Vertrauensbekundungen durch den linken Flügel der SPD nach der Ankündigung der Vertrauensfrage (dazu auch unten IV. 3. c.). Dass die im Mehrheitsvotum bemühten »Tatsachen« selbst unter Berücksichtigung des dem Kanzler eingeräumten Einschätzungsspielraums den Wegfall der politischen Handlungsfähigkeit nicht hinreichend belegen können, wird der Sache nach denn auch vom BVerfG anerkannt, wenn es den Wegfall der politischen Handlungsfähigkeit zu einem wesentlichen Teil darauf stützt, dass der Kanzler »hinreichend deutlich ausgedrückt (habe), dass er aus partei- und fraktionspolitischer Rücksichtnahme nicht Ross und Reiter nennt. Zusätzlich (habe) er mit dem Gewicht seiner politischen Vertrauenswürdigkeit von Erfahrungen berichtet, die er auch mit der Unaufrichtigkeit Einzelner aus dem eigenen politischen Lager, deren Namen er im dunkeln lässt, gemacht hat « ${ }^{28}$. Auf derselben Linie liegt es ferner, wenn in dem Mehrheitsvotum auf ein von den Antragstellern »nicht in Abrede gestelltes Gespräch «- wie sollten sie dieses vertrauliche Gespräch auch in Abrede stellen können - zwischen dem Bundeskanzler und dem Partei- und Fraktionsvorsitzenden Müntefering verwiesen wird, in dem Müntefering dem Kanzler bereits vor der Landtagswahl in Nordrhein-Westfalen mitgeteilt hatte, dass er Sorge »um die Handlungsfähigkeit« seiner Partei und letztlich auch der Bundesregierung habe ${ }^{29}$. Die Vorkommnisse, auf welche diese Beurteilung gegründet wurde, wurden auch hier nicht benannt. Deshalb blieb unklar, ob sich diese Sorgen nur auf aus der Tagespresse oder dem Rundfunk bereits bekannte kritische Äußerungen einzelner Mitglieder der Regierungsfraktionen sowie sonstiger Parteimitglieder stützten, auf die in dem durch das Bundeskanzleramt vorgelegten Dossier Bezug genommen wurde,

26 BVerfG, a.a.O. (Fn. 12), Rdnr. 161 = NJW 2005, S. 2669 (2673 f.).

27 S. dazu Minderheitsvotum Jentsch, BVerfG, a.a.O. (Fn. 12), Rdnr. 195 = NJW 2005, S. 2669 (2677).

28 BVerfG, a.a.O. (Fn. 12), Rdnr. $169=$ NJW 2005, S. 2669 (2674).

29 BVerfG, a.a.O. (Fn. 12), Rdnr. 171 = NJW 2005, S. 2669 (2674 f.). 
oder auf weitere nicht publik gewordene Umstände, welche einen Schluss auf eine Gefährdung der politischen Handlungsfähigkeit der Regierung zuließen.

\section{b) Der Bruch mit sonst allgemein anerkannten Kontrollgrundsätzen als Gefährdung der Normativität der Verfassung}

Mit diesem Verzicht auf eine Überprüfung der Sachverhalts, auf welchen sich die politische Beurteilung stützt, weicht das BVerfG von den sonst anerkannten Grundsätzen ab, die bezüglich der gerichtlichen Überprüfung von Entscheidungen staatlicher Organe bestehen, denen in bezug auf das Vorliegen von Entscheidungsvoraussetzungen ein Beurteilungsspielraum eingeräumt ist. In sonstigen Fällen der Zuerkennung von Beurteilungsspielräumen wird nämlich stets davon ausgegangen, dass das Gericht zu prüfen berechtigt (ja verpflichtet) ist, ob der der Beurteilung zugrunde liegende Sachverhalt zutreffend ermittelt wurde ${ }^{30}$. Diese Notwendigkeit der Sachverhaltsermittlung ist eine unerlässliche Voraussetzung jeder gerichtlichen Kontrolle. Sie ist dieser letztlich wesensimmanent. Ohne sie lässt sich die Normativität des Rechts nicht sichern. Eine Preisgabe dieses Grundsatzes bei einer Prüfung der Einhaltung verfassungsrechtlicher Vorgaben impliziert einen Verlust der Normativität der Verfassung. Bedenkt man die Bedeutung, die der Verfassung als einheitsstiftendem und integrierendem Fundament staatlicher Ordnung zukommt, so wiegt der Verlust ihrer Normativität besonders schwer. Er provoziert hier ganz spezifische Bedenken. Zwar ist die Verfassung nur eine Rahmenordnung ${ }^{31}$ und kein detailliertes und konkretisiertes Regelwerk wie einfachgesetzliche Bestimmungen. Das unterscheidet sie etwa von der in diesem Zusammenhang zur Illustration von Unterschieden oft bemühten Straßenverkehrsordnung. Auch verbietet sich eine in der Verfassungsgerichtsbarkeit zuweilen feststellbare Tendenz, die Verfassung mit einfachgesetzlichen Bestimmungen aufzuladen, wie sie insbesondere durch die zu starke Betonung des Systemgedankens im Verfassungsrecht begünstigt wird ${ }^{32}$. Soweit aber verfassungsrechtliche, den politischen Prozess leitende Vorgaben anerkannt sind und insofern einen verbindlichen Rahmen konstituieren, bedürfen sie strikter Beachtung. Dies schließt nicht aus, dass insoweit Beurteilungsspielräume anzuerkennen sind, die der Eigenart des Politischen Rechnung tragen. Unverzichtbar muss aber die Sachverhaltsaufklärung als notwendige Voraussetzung einer gerichtlichen Beurteilung bleiben. Deshalb darf es dem BVerfG nicht erlaubt sein, den für die Stellung der unechten Vertrauensfrage durch den Bundeskanzler maßgeblichen Sachverhalt auch nur teilweise aus seiner gerichtlichen Überprüfung auszuklammern. Eine solche Rücknahme der verfassungsgerichtlichen Kontrolle lässt sich

30 Dazu Hartmut Maurer, Allgemeines Verwaltungsrecht, 15. Aufl. 2004, \ 7, Rdnr. 58;

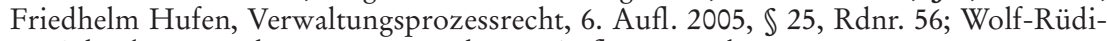
ger Schenke, Verwaltungsprozessrecht, 10. Aufl. 2005, Rdnr. 774.

31 Zur Verfassung als Rahmenordnung Ernst-Wolfgang Böckenförde, Die Methoden der Verfassungsinterpretation - Bestandsaufnahme und Kritik, NJW 1976, S. 2089 (2099).

32 Dazu Schenke, Verfassungsgerichtsbarkeit und Fachgerichtsbarkeit, 1987, S. 32 ff.; Rainer Wahl, Der Vorrang der Verfassung, Der Staat Bd. 20 (1981), S. 485 ff. 
auch nicht damit rechtfertigen, dass dem Kanzler gegenüber abgegebene, von ihm nicht offenbarte Äußerungen, welche ihn zum Schluss einer mangelnden politischen Handlungsfähigkeit veranlassten, ihrerseits wiederum Gegenstand politischer Bewertungen waren. Derartige Äußerungen legitimieren es noch nicht, auf ihre Benennung zu verzichten und ihr Vorliegen aus der gerichtlichen Überprüfung vollständig auszuklammern, wie dies vom BVerfG vertreten wird. Sie hindern das BVerfG nur daran, ihre Rationalität zu überprüfen, da es auf die Gründe, aus denen heraus das Vertrauen zum Bundeskanzlers entfallen ist, für die Beurteilung des Bestehens einer Vertrauenslage und einer ausreichenden politischen Unterstützung naturgemäß nicht ankommen kann.

Der Ausschluß einer gerichtlichen Überprüfung von Umständen, auf welche Kanzler und Fraktionsvorsitzender ihre Beurteilung der politischen Handlungsfähigkeit des Bundeskanzlers stützten, lässt sich auch nicht mit dem Hinweis des BVerfG ${ }^{33}$ in Frage stellen, »das Grundgesetz (habe) nur die Kontrolle politischer Herrschaft gewollt und nicht die Verrechtlichung des politischen Prozesses«. Die Erstreckung der verfassungsgerichtlichen Überprüfung auf das Vorliegen aller Umstände, die den Kanzler veranlassten, von einem Verlust seiner politischen Handlungsfähigkeit auszugehen und deshalb eine unechte Vertrauensfrage zu stellen, dient in den Fällen einer verdeckten Minderheitslage ausschließlich dem Zweck sicherzustellen, dass die vom BVerfG aufgestellte Forderung nach dem Bestehen rechtlicher Voraussetzungen für die Stellung einer unechten Vertrauensfrage in einer praktikablen Weise gesichert wird. Das trägt - wie oben schon angedeutet - dem Umstand Rechnung, dass es nicht angeht, einerseits verfassungsrechtlich materielle Anforderungen an die Stellung einer Vertrauensfrage $\mathrm{zu}$ postulieren, andererseits diese aber prozessrechtlich durch eine - auch bei Anerkennung von Beurteilungsspielräumen unserem Prozessrecht sonst nicht bekannte - Einschränkung der gerichtlichen Überprüfung in bezug auf eine »verdeckte Minderheitssituation des Bundeskanzlers « zur Bedeutungslosigkeit zu verurteilen. Die vom BVerfG zur Begründung seiner Prüfungsrestriktion angeführte »vom Grundgesetz gewollte Balance zwischen effektiver rechtlicher Bindung der öffentlichen Gewalt und der Ermöglichung wirksamer politischer Handlungsfreiheit « ${ }^{34}$ spricht damit gerade gegen die von ihm vertretene Ansicht, unter deren Zugrundelegung sich keine effektive gerichtliche Kontrolle des Bundeskanzlers ergeben würde. Auch wenn man der hier vertretenen Ansicht folgt, »bliebe dem Bundeskanzler ein eigener Einschätzungsspielraum in Bezug auf die festgestellten Tatsachen, insbesondere in Bezug auf deren Bedeutung für die zukünftige politische Entwicklung «, und würde »dem politischen Willensbildungsprozess mit seinen zulässigen, auch von taktischen und strategischen Rücksichtnahmen geprägten Verhaltensweisen und Rücksichtnahmen ... kein Schaden zugefügt « ${ }^{35}$.

33 BVerfG, a.a.O. (Fn. 12), Rdnr. $160=$ NJW 2005, S. 2669 (2673).

34 BVerfG, a.a.O. (Fn. 12), Rdnr. 154 = NJW 2005, S. 2669 (2672 f.).

35 S. auch BVerfG, a.a.O. (Fn. 12), Rdnr. 154 = NJW 2005, S. 2669 (2672 f.). 
Die Rücknahme der gerichtlichen Kontrolle hinsichtlich der eine »verdeckte Minderheitssituation des Bundeskanzler « begründenden Umstände erweckt schließlich auch noch insoweit Bedenken, als in ihrer Konsequenz sogar die Einhaltung solcher verfassungsrechtlicher Begrenzungen einer unechten Vertrauensfrage gefährdet erscheint, die durch die neue Entscheidung des BVerfG nicht in Frage gestellt wurden. Das betrifft die Fälle, bei denen es um die vorzeitige Herbeiführung eines dem Bundeskanzler als günstig erscheinenden Wahltermins ${ }^{36}$ oder die Delegitimierung des Bundesrats ${ }^{37}$ geht und bei denen auch das BVerfG von der Verfassungswidrigkeit einer derart motivierten unechten Vertrauensfrage ausgeht. Die Anerkennung einer »verdeckten Minderheitssituation «, bei der die für die Behauptung einer politischen Handlungsunfähigkeit maßgeblichen Umstände verdeckt und ungeprüft bleiben, lädt dazu ein, mit Scheinargumenten zu verdecken, dass die Auflösung des Bundestags tatsächlich aus verfassungsrechtlich unzulässigen Gründen betrieben wird. Es dürfte jedenfalls auf der Hand liegen, dass kein Bundeskanzler die Vertrauensfrage mit einer nach Ansicht des BVerfG und der h. M. unzulässigen Begründung stellen, sondern sich um eine verfassungs(gerichts)feste Begründung bemühen wird. Hier liefert ihm die neue Rechtsprechung wesentliche Hilfe und leistet damit (ungewollt) einer weiteren Schwächung der Normativität der Verfassung Vorschub.

\section{c) Die Veränderung des Kräfteverbältnisses zwischen Bundeskanzler und Bundestag als Konsequenz der Einschränkung der gerichtlichen Überprüfungsbefugnis}

\section{aa) Kritik und Vertrauen}

Eine Legitimation der Vertrauensfrage durch Stützung auf einen nach außen hin nicht offenbar gemachten Verlust der politischen Handlungsfähigkeit des Kanzlers, wie sie das BVerfG für möglich hält, provoziert aber auch insoweit schwerwiegende Bedenken, als sie tendenziell das Kräfteverhältnis zwischen Bundeskanzler und Bundestag erheblich verschiebt und zu einer beträchtlichen Stärkung der Stellung des Bundeskanzlers gegenüber dem Bundestag führt. Dies birgt die Gefahr in sich, dass in Gesprächen mit dem Bundeskanzler geäußerter Kritik in Verbindung mit anderen (für sich gesehen jedoch noch nicht ausreichenden) Umständen eine ausschlaggebende Bedeutung für den Wegfall des politischen Vertrauens beigemessen wird. Das ist aber damit unvereinbar, dass es für das Vorliegen eines Vertrauens wie am Beispiel der echten Vertrauensfrage deutlich wird - letztlich immer nur auf die nach außen hin erklärte oder jedenfalls zu erwartende Zustimmung zu Person und Politik des Kanzlers ankommen kann. Aus welchen Gründen diese Zustimmung erfolgt, ist irrelevant. Das ergibt sich nicht nur daraus, dass die Feststellung eines bestehenden Vertrauens sich in gewissem Umfang - selbst nach Ansicht des

36 BVerfG, a.a.O. (Fn. 12), Rdnr. 137 = NJW 2005, S. 2669 (2671) und schon früher BVerfGE 62, S. 1 (42 f.).

37 BVerfG, a.a.O. (Fn. 12), Rdnr. 151 = NJW 2005, S. 2669 (2672). 
BVerfG - zumindest auch an nach außen hin zutage tretenden Umständen (» Tatsachen «) zu orientieren hat. Es folgt auch daraus, dass es sich bei dem Begriff des Vertrauens um keine moralische, sondern - wie der Kanzler ${ }^{38}$ in seiner Begründung der Vertrauensfrage insoweit zu Recht hervorhob - um eine politische Kategorie handelt. Vertrauen i. S. des Art. 68 GG kann man demgemäß selbst dann haben, wenn man politische Auffassungen des Kanzlers nicht teilt, ja diese heftig kritisiert, trotzdem aber bereit ist, diesen letztlich zu unterstützen, $d$. h. für ihn und sein Programm bei den Entscheidungen im Bundestag zu votieren. Dabei kann ein solches Votum auf den unterschiedlichsten Motiven beruhen, etwa darauf, dass man für die eigenen politischen Auffassungen keine Realisierungschance sieht, dass man die eigene Partei und die durch sie gestellte Regierung nicht schwächen will, dass die Regierungspolitik im Verhältnis zur Oppositionspolitik als kleineres Übel erscheint, dass man eine Vorverlegung von Neuwahlen wegen eines hier zu befürchtenden negativen Ausgangs vermeiden will oder dass man befürchtet, nicht mehr als Kandidat für die nächste Bundestagswahl aufgestellt oder gar politisch geächtet zu werden. Kann damit aber Vertrauen i. S. des Art. 68 GG trotz einer kritischen Einstellung zur Politik des Bundeskanzlers bestehen, so darf eine nach außen nicht in Erscheinung tretende und insoweit verdeckte Kritik, die nur gegenüber dem Bundeskanzler oder jedenfalls nur fraktions- oder parteiintern zum Ausdruck gebracht wird, bei einer verdeckten Minderheitssituation keine ausschlaggebende Bedeutung für die Zulässigkeit einer unechten Vertrauensfrage beigemessen werden, ohne dass die vom Kanzler geltend gemachten und für den Verlust seiner Handlungsfähigkeit angeführten verdeckten Umstände einer (unter Beachtung des Beurteilungsspielraums des Kanzlers) durchzuführenden verfassungsgerichtlichen Kontrolle unterworfen werden.

So mutet es denn auch einigermaßen seltsam, ja widersprüchlich an und entbehrt nicht einer gewissen Pikanterie, wenn einerseits das BVerfG ${ }^{39}$ durchaus zu Recht betont, dass die Aufgabe der Kontrolle keineswegs ausschließlich der parlamentarischen Opposition zufalle, sondern auch den Abgeordneten der Regierungsfraktionen, hier allerdings regelmäßig lediglich partei- und fraktionsintern geäußert werde, gleichzeitig aber das Gericht durch Verneinung einer gerichtlichen Kontrollmöglichkeit keinerlei prozedurale Vorsorge dagegen trifft, dass eben diese für das parlamentarische Regierungssystem essentielle interne Kritik, durch den Bundeskanzler zum Anlass genommen wird, mittels einer unechten Vertrauensfrage auf die Auflösung des Bundestags hinzuwirken. Dass die Befürchtung durchaus real erscheint, dass eine verfehlte Einschätzung der politischen Mehrheitsverhältnisse durch den Bundeskanzler und der hiermit einhergehende verfassungswidrige Einsatz der unechten Vertrauensfrage der gerichtlichen Kontrolle entgleitet, wird an dem Dossier deutlich, das das Bundeskanzleramt zur Legitimation der Vertrauensfrage nachgeschoben hat. Dieses belegte zwar die ohnehin schon allgemein bekannte Tatsache, dass eine Reihe von Abgeordneten der Regierungsfraktionen der Reformpolitik des

38 Stenografischer Bericht, a.a.O., S. 17467 (B).

39 BVerfG, a.a.O. (Fn. 12), Rdnr. 134 = NJW 2005, S. 2669 (2670 f.). 
Bundeskanzlers kritisch gegenüberstanden. Diese Kritik - die bereits im Vorfeld der Verabschiedung von Hartz-IV in den Medien vernehmbar war, die Kritiker aber bezeichnenderweise nicht daran hinderte, dennoch nahezu geschlossen (bei einer Stimmenthaltung) für dieses Gesetzesprojekt zu stimmen - ließ aber in keiner Weise den Schluß zu, es habe dem Kanzler an der erforderlichen Zustimmung zu seiner Politik gemangelt $t^{40}$. Nichts anderes kann dann aber für eine nicht öffentlich, sondern nur gegenüber dem Kanzler verdeckt geäußerte Kritik gelten. Beide Male muss die Einschätzung des Bundeskanzlers durch das BVerfG darauf überprüfbar sein, ob sie eindeutig fehlerhaft ist, was aber ohne eine Ermittlung der Beurteilungsbasis nicht möglich ist. Deren Aufhellung war um so unerlässlicher, als eine Vermutung dafür spricht, dass dieselben Gründe, welche den Kanzler dazu veranlassten, in öffentlich geäußerter Kritik ein Zeichen für den Verlust seiner politischen Handlungsfähigkeit zu sehen ${ }^{41}$, ihn auch dazu bewogen, intern geäußerte Kritik als Zeichen des Verlusts der politischen Handlungsfähigkeit zu bewerten.

\section{bb) Vertrauensbekundungen und Vertrauen}

Das Abstellen auf eine nicht offen zutage tretende, durch das BVerfG insoweit nicht nachprüfbare Minderheitssituation wird noch problematischer, wenn man den Parlamentsabgeordneten die Möglichkeit nimmt, die Einschätzung des Bundeskanzlers durch eine nach der Ankündigung der unechten Vertrauensfrage abgegebene Vertrauensbekundung zu widerlegen. Eben dies geschieht aber, wenn solche offen zutage getretenen Vertrauensbekundungen ${ }^{42}$ durch den Bundeskanzler - wie dies in der Begründung seiner Vertrauensfrage geschah - als »unehrlich« abgetan werden und

40 Die Drohung eines einzelnen Abgeordneten der Regierungsfraktionen, des Abgeordneten Schreiner, aus der SPD auszutreten, vermochte die Einschätzung des Verlusts der politischen Mehrheit nicht zu tragen, zumal dieser auch ohne eine Änderung des politischen Kurses der Regierung sich schon bald dahingehend erklärte, weiter in der SPD zu bleiben, und er bei der Neuwahl des Bundestags durch seine Partei sogar als Spitzenkandidat im Saarland aufgestellt wurde. Den in dem Dossier wiedergegebenen Stimmen aus dem außerparlamentarischen Raum (wie die des früheren SPD-Vorsitzenden Lafontaine) kam für die Beurteilung der im Rahmen des Art. 68 GG einzig maßgeblichen politischen Mehrheitsverhältnisse im Bundestag ohnehin keine Bedeutung zu bzw. konnte nur bei Zulassung einer plebiszitär-demokratischen Verformung des bewusst repräsentativ-demokratischen Systems des GG Relevanz erlangen.

41 Bezeichnenderweise spielt denn auch das Dossier und die dort enthaltenen Stellungnahmen bei der Beurteilung der politischen Handlungsfähigkeit keine Rolle, was nur dann verständlich ist, wenn man annimmt, dass sie nach Auffassung des BVerfG noch nicht ausreichten, um die Annahme einer politischen Handlungsunfähigkeit des Kanzlers hinreichend $\mathrm{zu}$ belegen und deshalb auf vom Bundeskanzler behauptete, nicht offen zutage getretene, durch das Gericht nicht überprüfbare Umstände abgestellt wurde.

42 So äußerte etwa die Parteilinke Nahles im ZDF: »Ich sage ganz klar, dass die Linken in der Fraktion dem Kanzler auf jeden Fall ihr Vertrauen aussprechen. Und er hatte auch in den letzten Jahren immer Mehrheiten für seine Politik und das wird auch so bleiben«, s. DPA/Reuthers, Artikel vom 6.06.2005, auf http://www.stern.de/politik/deutschland/ 541345.htm.?nv=?pr\&pr=). Der SPD-Abgeordnete Michael Müller und Nahles betonten an dieser Stelle ferner, auch im SPD-internen Streit um die Reform der Unternehmensbesteuerung würden die linken Kritiker der Reform loyal zu Schröder stehen. 
diese Einschätzung durch das BVerfG ungeprüft übernommen wird. Damit wird nicht ausreichend beachtet, dass bereits die Ankündigung einer unechten Vertrauensfrage eine ähnliche Wirkung entfalten kann wie die Stellung einer Vertrauensfrage. Die drohende vorzeitige Auflösung des Bundestags mit ihren Folgen für den einzelnen Bundestagsabgeordneten, aber auch für seine Partei kann für die gegenüber der Regierungspolitik kritisch eingestellten Abgeordneten durchaus einen Anlass bilden, trotz ihrer politischen Skepsis den Bundeskanzler zu unterstützen und ihm Vertrauen zu bekunden. Sie kann insoweit die durch Art. 68 GG bezweckte Stabilität der Regierung und ihre Handlungsfähigkeit zur Folge haben, zumal es dem Bundeskanzler bei solchen Vertrauensbekundungen durchaus zumutbar und ohne politische Beschädigung möglich ist, von der angekündigten unechten Vertrauensfrage - im Einklang mit der Teleologie des Art. 68 GG - Abstand zu nehmen. Diese Stabilisierungsfunktion wird nun aber vereitelt, wenn man der politischen Vertrauensbekundung keinerlei Bedeutung beimisst, gleichzeitig aber dem Abgeordneten die Möglichkeit nimmt, dem Kanzler im Rahmen einer echten Vertrauensfrage sein Vertrauen auszusprechen und damit das Integrations- und Stabilitätspotential der echten Vertrauensfrage zu nutzen. Eine Vertrauenserklärung im Rahmen einer unechten Vertrauensfrage vermag diese Stabilisierungswirkung naturgemäß nicht zu entfalten, da das Scheitern einer solchen Vertrauensfrage vorprogrammiert ist, weil es hier bei knappen Mehrheitsverhältnissen bereits genügt, wenn einige wenige Abgeordnete der Regierungsfraktionen sich bei der Vertrauensfrage dem Wunsche des Kanzlers gemäß der Stimme enthalten.

\section{cc) Kanzlerdemokratie und Verkehrung des parlamentarischen Regierungssystems}

Aus dem Gesagten ergibt sich zugleich, dass die verfassungsgerichtliche Tolerierung einer Vertrauensfrage bei einer vom Bundeskanzler behaupteten »verdeckten Minderheitssituation « ohne eine gerichtliche Überprüfbarkeit ihr zugrunde gelegter wesentlicher Fakten in der Tat das Verhältnis des Bundeskanzlers zum Bundestag und hier speziell auch zu den Regierungsfraktionen - in grundsätzlicher Weise verändert. Sie räumt dem Kanzler bei verdeckt geäußerter Kritik an seiner Politik die Möglichkeit zu einer Auflösung des Bundestags ein, ohne dass die Bundestagsabgeordneten, die bereit und willens sind, den Kanzler bei politischen Abstimmungen zu unterstützen, die Möglichkeit haben, sich einer Auflösung des Bundestags politisch zu widersetzen und ohne dass das BVerfG einer solchen Vorgehensweise einen Riegel vorzuschieben vermag. Die unechte Vertrauensfrage wirkt damit bei einer »verdeckten Minderheitssituation des Kanzlers« als ein Disziplinierungsmittel, mit dessen Hilfe innerparteiliche Demokratie und die durch Art. 38 Abs. 1 S. 2 GG garantierte Freiheit und Unabhängigkeit des Abgeordneten untergraben und die Machtbalance zwischen Bundeskanzler und Bundestag empfindlich gestört werden kann. Die ohnehin schon starke Stellung des Bundeskanzlers gegenüber den Regierungsfraktionen und ihren Mitgliedern wird erheblich ausgebaut und ein weiterer Schritt in die Richtung einer Kanzlerdemokratie getan. Der Sache nach bedeutet die bundesverfassungsgerichtliche Judikatur zur »verdeckten Minderheitssituation des Minderheitskanzlers « nämlich nichts anderes als die de facto-Einführung eines Auf- 
lösungsrechts des Bundeskanzlers, wie es in der konstitutionellen Monarchie dem Monarchen, in der Weimarer Republik dem Reichspräsidenten (nicht dem Reichskanzler!) zustand. Durch Behauptung einer verdeckten, durch das BVerfG nicht überprüfbaren Minderheitssituation vermag der Bundeskanzler nämlich schon bei "gefühltem Misstrauen« auf eine Auflösung des Bundestags hinzuwirken und wird diese auch regelmäßig durchsetzen (s. dazu auch unten IV. 3. e.). Das parlamentarische Regierungsprinzip wird damit gewissermaßen auf den Kopf gestellt bzw. pervertiert: Nicht der Bundestag spricht dem Bundeskanzler sein Misstrauen aus, sondern der Bundeskanzler dem Parlament, indem er faktisch dessen Auflösung bewirkt.

\section{d) Geheimnisschutz als Rechtfertigung einer Einschränkung der gerichtlichen Überprüfung}

Die vom Mehrheitsvotum befürwortete Einschränkung der gerichtlichen Kontrolle durch eine in wesentlichen Punkten ungeprüfte Übernahme der politischen Lageeinschätzung des Bundeskanzlers lässt sich auch nicht im Hinblick auf Geheimhaltungsinteressen des Bundeskanzlers rechtfertigen. Dabei soll hier ganz davon abgesehen werden, dass im konkreten Fall die Berufung des BVerfG auf ein Geheimhaltungsinteresse schon deshalb fragwürdig erschien, weil hier das Bundeskanzleramt in einem dem Bundespräsidenten, den Regierungsfraktionen und dem BVerfG vorgelegten, auf Presse- und Rundfunkberichten beruhenden Dossier die Kritiker des Bundeskanzlers aus dem Regierungslager benannt und damit - in der Terminologie des BVerfG ausgedrückt - »Ross und Reiter « offenbart hatte, so dass insofern ein Geheimhaltungsinteresse bezüglich dieser Personen nur schwerlich begründbar war. Die Berufung auf Geheimhaltungsinteressen vermag nämlich ganz losgelöst von dem entschiedenen Fall als Grund für die Einschränkung der bundesverfassungsgerichtlichen Kontrolle allgemein nicht zu überzeugen. Zum einen besteht nämlich auch dann, wenn man die vom BVerfG befürwortete Restriktion seiner Überprüfungsbefugnis ablehnt, für den Bundeskanzler keine Verpflichtung, von ihm als geheimhaltungsbedürftig eingestufte Umstände zu offenbaren (aa.), zum anderen würden sich, selbst wenn man solche Offenbarungspflichten befürwortete, prozessuale Handhaben anbieten, um einem Geheimhaltungsinteresse Rechnung zu tragen (bb.).

a) Die Offenlegung der für die unechte Vertrauensfrage maßgeblichen verdeckten Umstände als Obliegenheit

Schon in der mündlichen Verhandlung vor dem BVerfG wurde durch mich vorgetragen, dass den vom BVerfG betonten Geheimhaltungsinteressen des Bundeskanzlers ohne Bruch mit prozessualen Grundsätzen dadurch Rechnung getragen werden kann, dass man den Bundeskanzler nicht als verpflichtet ansieht, entsprechende Umstände, die ihn zur Annahme des weggefallenen des Vertrauens veranlassten, zu offenbaren. Deren Nichtoffenlegung bedeutet allerdings - und hierin liegt ein wesentlicher Unterschied zum BVerfG - , dass ihnen keine ausschlaggebende Bedeu- 
tung für die gerichtliche Überprüfung zukommen kann. Es handelt sich hierbei also juristisch gesehen um eine Obliegenheit. Es besteht mithin keine Verpflichtung des Bundeskanzlers, die Umstände zu benennen, auf die er den Vertrauensverlust stützt. Wenn er sie aber nicht benennt, hat dies für ihn die nachteilige Folge, dass er dann auf solche nicht belegte Umstände den behaupteten Vertrauensverlust nicht stützen kann. Gerade dann, wenn man sich den exzeptionellen Charakter der unechten Vertrauensfrage vor Augen hält - wie er darin zum Ausdruck kommt, dass diese auch nach dem BVerfG (anders als eine echte Vertrauensfrage) an besondere Voraussetzungen gebunden ist - , kann man von dem Bundeskanzler in der Tat verlangen, dass er zur Begründung eines auf andere Weise noch nicht ausreichend belegten Vertrauensverlusts »Ross und Reiter « nennt. Ohne die Annahme einer entsprechenden Obliegenheit könnte er nämlich ohne jede politische Beschädigung eine unechte Vertrauensfrage stellen, so dass sich diese - zumal wenn ihm erlaubt wird, die Vertrauensfrage mit der Notwendigkeit einer Anrufung des demokratischen Souveräns $\mathrm{zu}$ verbinden - von einem Notbehelf zur Bewältigung einer politischen Krisenlage in ein taktisches Instrument im politischen Machtkampf verwandelte. Es spielte dann bei seinem Einsatz auch keine Rolle, dass opponierende Abgeordnete der Regierungsfraktionen ohne die Stellung einer unechten Vertrauensfrage den Bundeskanzler und seine Politik trotz ihrer politischen Vorbehalte bei entscheidenden Abstimmungen oftmals politisch unterstützt haben und ihm bei einer echten Vertrauensfrage ihr Vertrauen ausgesprochen hätten, um die Stabilität und Handlungsfähigkeit der Regierung zu sichern, aber auch um sich einem politischen Druck und politischen Sanktionen durch ihre Partei aber auch durch die öffentliche Meinung zu entziehen. Diese Stabilitätsmechanismen kämen bei einer unechten Vertrauensfrage, die sich auf politische Vorgänge stützte, die im Verborgenen blieben und weder der Partei, noch der Öffentlichkeit bekannt würden, nicht zum Tragen.

\section{bb) Prozessuale Handhaben zur Sicherung geheimhaltungsbedürftiger Umstände} Im übrigen ließe sich selbst dann, wenn man ein Geheimhaltungsinteresse des Bundeskanzlers hinsichtlich der Umständen bejahte, auf die er seine politische Einschätzung stützt, diesem Bedürfnis durch eine Ausgestaltung des Verfahrens Rechnung tragen, die einerseits Geheimhaltungsinteressen des Kanzlers wahrt, andererseits aber doch bezüglich der geheimhaltungsbedürftigen Umstände nicht jede Prüfung ausschließt. In Betracht käme insoweit ein in-camera-Verfahren, wie es vom BVerfG ${ }^{43}$ sonst dort, wo es bei der gerichtlichen Entscheidung von Streitigkeiten auf Umstände ankommt, hinsichtlich derer ein Geheimhaltungsinteresse besteht, als verfassungsrechtlich gefordert angesehen wurde. Entgegenstehende einfachgesetzliche prozessuale Regelungen hat das BVerfG als verfassungswidrig

43 BVerfGE 101, S. 106 ff. Zu der aufgrund dieser Entscheidung erfolgten Novellierung des $\ 99$ VwGO s. Ferdinand Kopp / Wolf-Rüdiger Schenke, Verwaltungsgerichtsordnung, Kommentar, 14. Aufl. 2005, $\mathbb{9} 99$, Rdnrn. 2 ff.; Richard Rudisile, in: Friedrich Schoch / Eberhard Schmidt-Aßmann / Rainer Pietzner, Verwaltungsgerichtsordnung, Kommentar, 2005, \$99, Rdnrn. 4 ff. 
verworfen. Gründe, aus denen heraus sich die Übertragung dieser Rechtsprechung auf die gerichtliche Kontrolle von Organakten im Rahmen einer Organstreitigkeit verbieten, sind nicht ersichtlich. Zumindest hätten diese aber durch das BVerfG dargelegt werden müssen.

\section{e) Die Kontrolle durch andere Verfassungsorgane als Substitution der verfassungsgerichtlichen Kontrolle}

Die vom BVerfG ${ }^{44}$ zur Rechtfertigung seines judicial self-restraint beschworenen Kontrollmechanismen, mit deren Hilfe die anderen Verfassungsorgane in die Lage versetzt sein sollen, eine Auflösung des Bundestags zu verhindern, vermögen ein solches (faktisches) Parlamentsauflösungsrecht letztlich nicht in Frage zu stellen. Diese Kontrollmechanismen bestehen zwar de iure, sie sind aber aus verschiedenen Gründen de facto nicht wirksam. Es sollte keiner besonderen Hervorhebung bedürfen, dass der Bundeskanzler, der eine Bundestagsauflösung anstrebt, keine Gewähr für eine wirksame Kontrolle der von ihm selbst angestrengten unechten Vertrauensfrage bietet. Aber auch eine Kontrolle durch den Bundestag und durch den Bundespräsidenten wird regelmäßig defizitär ausfallen. $\mathrm{Da}$ von der Opposition, die einen Regierungswechsel anstrebt, schwerlich ein Vertrauensvotum für den Bundeskanzler erwartet werden kann - zumal dieses vom politischen Gegner entsprechend ausgenutzt und als Zeichen politischer Schwäche gedeutet werden könnte - genügten bereits die Stimmen von wenigen Abgeordneten der Regierungsfraktionen, um die Vertrauensfrage »scheitern« zu lassen. Regelmäßig dürfte aber die Zahl der Abgeordneten aus den Regierungsfraktionen, die dem Kanzler wunschgemäß kein Vertrauen aussprechen, weit höher ausfallen. Ein Vertrauensvotum für den Kanzler mit dem Ziel, die spätere Auflösung zu verhindern, wäre nämlich in Wahrheit kein Votum für, sondern ein Votum gegen den Bundeskanzler, das dessen Stellung schwächen müsste, wenn es dennoch später oder auf andere Weise zu einer neuen Bundestagswahl kommen würde ${ }^{45}$. Insoweit trifft die Aussage des Partei- und des Fraktionsvorsitzenden Münteferings, man könne dem Bundeskanzler auch dadurch sein Vertrauen aussprechen, dass man sich bei der Abstimmung über die Vertrauensfrage der Stimme enthalte ${ }^{46}$, so paradox sie auch auf den ersten Blick anmuten mag, genau den Nagel auf den Kopf. Gerade der Kanzler, der sich aufgrund der gegebenen politischen Situation bei vernünftiger Würdigung der Sachlage seiner politischen Mehrheit sicher sein könnte, darf gewiß sein, dass das mit der unechten Vertrauensfrage erstrebte Ziel nicht am Stimmverhalten der Abgeordneten aus dem

44 So Leitsatz 4 c der Entscheidung des BVerfG, a.a.O. (Fn.12), Rdnrn. 155 ff., 160 = NJW 2005, S. 2669 (2673) und schon früher BVerfGE 62, S. 1 (3, 51).

45 Realistischerweise ausgeschlossen - da auf eine Demontage des Kanzlers hinauslaufend - ist ohnehin ein positives Vertrauensvotum durch die Mehrheit der Mitglieder der Regierungsfraktionen, das sich darauf stützt, dass der Kanzler die politischen Mehrheitsverhältnisse eindeutig fehlerhaft eingeschätzt und sich deshalb verfassungswidrig verhalten habe. www2.onnachrichten.t-online.de/dyn/c/46/64/17/4664174.html. 
Regierungslager scheitern wird. Der für die moderne Parteiendemokratie charakteristische politische Gleichklang zwischen der Bundesregierung und einer ihr verbundenen Parlamentsmehrheit findet hier einmal mehr seine Bestätigung.

Aber auch der Bundespräsident vermag keine wirksame Kontrolle auszuüben. Die Zulassung einer Vertrauensfrage bei einer verdeckten Minderheitssituation, deren Vorhandensein durch den Bundeskanzler nicht weiter belegt werden muss, macht es nicht nur dem BVerfG, sondern auch dem Bundespräsidenten unmöglich, als »Hüter der Verfassung « zu fungieren. Ansatzpunkte für eine dem Bundeskanzler gegenüber dem Bundespräsidenten obliegende weitergehende Offenbarungspflicht hinsichtlich des nicht offen zutage tretenden politischen Sachverhalts, auf den er seine politische Einschätzung stützt, sind dem Urteil des BVerfG nicht zu entnehmen. Damit wird dem Bundespräsidenten die Möglichkeit genommen, unter Berufung auf die ihn nach Art. 68 GG treffende verfassungsrechtliche Prüfungspflicht und damit losgelöst von einer politischen Bewertung des Auflösungsverlangens die Auflösung des Bundestags zu verhindern. Es bleibt allerdings die ihm nach h. M. de iure eingeräumte Befugnis, ein politisches Ermessens in bezug auf die Auflösung auszuüben. Realistischerweise ist freilich hier - wie gerade die Vorgänge in Verbindung mit der unechten Vertrauensfrage Schröders belegen - die Möglichkeit des Bundespräsidenten, dem Auflösungsverlangen ein politisch motiviertes Votum entgegenzusetzen, gelinde ausgedrückt sehr begrenzt. Bei einer unechten Vertrauensfrage werden nämlich die im Bundestag vertretenen Parteien regelmäßig, wenn auch oft unterschiedlich motiviert, dem Auflösungsverlangen beipflichten bzw. sich diesem jedenfalls nicht offen widersetzen. Auch die Bevölkerung und die öffentliche Meinung werden einem Neuwahlwunsch regelmäßig positiv gegenüberstehen. Dies nicht nur deshalb, weil hier dem demokratischen Souverän eine zusätzliche Möglichkeit eingeräumt wird, durch ein außerordentliches Votum politische Fragen zu entscheiden, sondern auch deshalb, weil ein übereinstimmender Wunsch der im Bundestag vertretenen politischen Parteien regelmäßig auf die öffentliche Meinung zurückwirkt und, wenn hier nicht ohnehin bereits ein solcher Wahlwunsch vorhanden ist, dieser jedenfalls durch die Parteien erzeugt wird. Deutlich wurde dies gerade auch in Verbindung mit der Vertrauensfrage Gerhard Schröders. Hatten sich hier unmittelbar vor der Wahl in Nordrhein-Westfalen bei einer demoskopischen Umfrage noch über $70 \%$ der Befragten auch für den Fall eines Regierungswechsels in Nordrhein-Westfalen gegen die Ansetzung von Neuwahlen ausgesprochen ${ }^{47}$, so schlug hier nach der am Wahlabend erfolgten Ankündigung von Neuwahlen durch den Bundeskanzler innerhalb kürzester Zeit die Stimmung um und wurde der Bevölkerung durch die politischen Parteien der Neuwahlwunsch »einprogrammiert «. Bei einer solchen Lage kann aber vom Bundespräsidenten, der von Amts wegen bei keiner politischen Partei verankert ist, dem auch die unmittelbare demokratische Legitimation fehlt und der auch im übrigen nach dem Grundgesetz nur eine schwache politische Stellung besitzt, schwerlich erwartet werden, dass er sich einem von

47 S. Bericht der Financial Times Deutschland vom 18.05.2005, ftd.de, wonach sich $71 \%$ der Bürger gegen Neuwahlen ausgesprochen hatten. 
allen Seiten an ihn herangetragenen Neuwahlwunsch widersetzen wird. Sind auch ein übereinstimmender Neuwahlwunsch von allen im Parlament vertretenen politischen Parteien sowie der dadurch erzeugte Wahlwunsch der Bevölkerung nach richtiger Ansicht für die Beurteilung der rechtlichen Voraussetzungen für eine Auflösung des Bundestags irrelevant, so kommt ihm doch um so größere politische Relevanz für die Ausübung des politischen Ermessens des Bundespräsidenten zu. Dem Bundespräsidenten ist es bei realistischer, nicht wirklichkeitsblinder Betrachtung politisch kaum möglich, sich hierüber hinwegzusetzen. Mit welchen nachvollziehbaren politischen Gründen soll sich der Bundespräsident bei einer solchen Situation einem von allen relevanten politischen Kräften an ihn herangetragenen Auflösungsverlangen entgegenstellen?

\section{Resümee}

Die angestellten Überlegungen dürften gezeigt haben, dass sich die Verfassungslandschaft seit dem 25.08.2005 verändert hat. Die Entscheidung des BVerfG hat nicht nur das repräsentativ-demokratische System und die Bundesstaatlichkeit geschwächt, sondern auch das Kräfteverhältnis zwischen Bundeskanzler und Bundestag erheblich verändert und einen wesentlichen Schritt in Richtung auf eine Kanzlerdemokratie vollzogen. Vor allem aber ist die Normativität der Verfassung in einem zentralen politischen Bereich in Frage gestellt und damit zugleich die Autorität des BVerfG in Mitleidenschaft gezogen worden. Eine große Chance, den im Volksmund verbreiteten bösen Spruch »Politik geht vor Recht « in einem spektakulären Fall zu widerlegen und das Verfassungsbewusstsein der Bürger zu stärken, wurde verspielt. Unter diesen Umständen wäre es in der Tat die ehrlichere und konsequentere - wenngleich im Ergebnis aber ebenfalls nicht befriedigende - Lösung gewesen, im Einklang mit dem in der Begründung abweichenden Votum der Verfassungsrichterin Lübbe-Wolff ${ }^{48}$ auf materiellrechtliche Bindungen des Bundeskanzlers bei der Ausübung der Vertrauensfrage überhaupt zu verzichten und nicht verfassungsrechtliche Postulate aufzustellen, die nur eine papierene Wirksamkeit entfalten.

\section{Zusammenfassung}

Die Entscheidung des Bundesverfassungsgerichts vom 25.08.2005, mit welcher die Verfassungsmäßigkeit der Auflösung des 15. Deutschen Bundestags bestätigt wurde, hat das Verfassungsgefüge des Grundgesetzes in tiefgreifender Weise verändert. Sie hält zwar - insoweit im Einklang mit der bisherigen Rechtsprechung - formal daran fest, daß die Stellung einer Vertrauensfrage nach Art. 68 GG mit dem Ziel, die Auflösung des Bundestags herbeiführen (sogenannte unechte Vertrauensfrage), verfassungsrechtlich nur dann zulässig ist, wenn die politische Handlungsfähigkeit des

48 BVerfG, a.a.O. (Fn. 12), Rdnrn. 213 ff. = NJW 2005, S. 2669 (2679 ff.). 
Bundeskanzlers nicht mehr gewährleistet ist. Die verfassungsgerichtliche Überprüfung dieser Rechtmäßigkeitsvoraussetzung wird aber nunmehr so weit zurückgenommen, daß in praxi für die Stellung einer unechten Vertrauensfrage kaum noch Hindernisse bestehen. Dadurch wird u. a. die Stellung des Bundeskanzlers im Verhältnis zum Bundestag erheblich gestärkt und ein weiterer Schritt in Richtung auf eine Kanzlerdemokratie getan.

\section{Summary}

The decision of the Federal Constitutional Court (Bundesverfassungsgericht) of August 25th 2005, by which the court declared the dissolution of the 15th German Bundestag to be constitutional, has changed the constitutional equilibrium fundamentally. Formally in coherence with former decisions the court has stated that a motion of the Federal chancellor for a vote of confidence (Art. 68 Basic Law), that is primarily directed towards the dissolution of the parliament (so-called pseudo call for a vote of confidence - unechte Vertrauensfrage), is only constitutional, if the chancellor has lost his political capacity to act. However, the court has significantly reduced the level of constitutional scrutiny, it applies in such cases. As a consequence there are also almost no legal barriers for a pseudo vote of confidence. Hereby the court has strengthened the position of the chancellor in relation to the parliament. This means a further step towards a chancellor dominated democracy. 